\title{
Modification of Indacenodithiophene-Based Polymers and its Impact on Charge Carrier Mobility in Organic Thin-Film Transistors
}

\author{
Andrew Wadsworth ${ }^{\dagger}$, Hu Chen ${ }^{\ddagger}$, Karl J. Thorley ${ }^{\dagger}$, Camila Cendras, Mark Nikolka", \\ Helen Bristow ${ }^{\dagger}$, Maximilian Moser $^{\dagger}$, Alberto Salleo ${ }^{\S}$, Thomas D. Anthopoulos ${ }^{\ddagger}$, \\ Henning Sirringhaus", Iain McCulloch* ${ }^{\star \dagger}$.
}

† Department of Chemistry and Centre for Plastic Electronics, Imperial College London, Exhibition Road, London, SW7 2AZ, United Kingdom.

‡ King Abdullah University of Science and Technology (KAUST), KAUST Solar Center (KSC), Physical Sciences and Engineering Division (PSE), Thuwal, 23955-6900, Saudi Arabia.

$\S$ Department of Materials Science and Engineering, Stanford University, 476 Lomita Mall, Stanford, CA 94305, USA.

II Cavendish Laboratory, University of Cambridge, J. J. Thompson Avenue, Cambridge CB3 oHE, United Kingdom.

KEYWORDS: OTFT, mobility, IDT-BT, p-type

\begin{abstract}
The polymer indacenodithiophene-co-benzothiadiazole (IDT-BT) has been thoroughly studied for its use in p-type organic thin-film transistors over the course of the last decade. Whilst a variety of modifications have been made to its structure, few analogues have been able to match or surpass the hole mobility that can be obtained by IDT-BT. Here, we discuss the rationale behind the chemical modifications that have been utilized and suggest design principles towards high mobility indacenodithiophene-based polymers. It is clear that planarizing intramolecular interactions, that exist between the peripheral thiophene of the IDT unit and the benzothiadiazole, are imperative for achieving high hole mobilities in this relatively amorphous polymer. Moreover, despite the less ordered backbones of the extended fused-ring cores that have recently been utilized (TIF-BT and TBIDT-BT), high mobilities were still attained in these polymers owing to additional interchain charge transfer. Thus, maintaining the beneficial thiophene - benzothiadiazole intramolecular interactions, whilst further extending the IDT core to promote interchain charge transfer is a logical strategy towards high mobility p-type polymers.
\end{abstract}

\section{INTRODUCTION}

The progress made in organic semiconducting polymers over the course of the last twenty years has played a pivotal role in the development of solution-processed electronics, particularly in organic thin-film transistors (OTFTs), organic solar cells (OSCs) and organic light-emitting diodes (OLEDs). ${ }^{1-4}$ The use of semiconducting polymers, in OTFTs, for example, has been explored extensively in order to create the next-generation of solution-processed electronics; for logic circuits and lightweight or flexible display applications. ${ }^{5-7}$ As such, a greater understanding of the strategic design principles required for high performance OTFT materials has become much more apparent in recent times. The charge carrier mobility $(\mu)$ can be considered as the speed ( $\mathrm{cm} \mathrm{s}^{-1}$ ) at which the charge carriers move in the material in a given direction under an applied electric field $\left(\mathrm{V} \mathrm{cm}^{-1}\right)$, and is widely used as the figure of merit when evaluating the performance of organic semiconductors in OTFTs. Over the course of the last decade, the charge carrier mobility of organic semiconducting polymers has improved dramatically, to over $1 \mathrm{~cm}^{2} \mathrm{~V}^{-1} \mathrm{~s}^{-1}$, as a result of optimized fabrication processes and rational design of organic semiconducting materials. Current state of the art p-type OTFTs are able to achieve mobilities of over $10 \mathrm{~cm}^{2} \mathrm{~V}^{-1} \mathrm{~s}^{-1}$, usually in blend systems, and mobilities of $5-10 \mathrm{~cm}^{2} \mathrm{~V}^{-1} \mathrm{~s}^{-1}$ are possible in $\mathrm{n}$ type OTFTs. ${ }^{8-13}$

There are four common OTFT architectures, summarized in Figure 1, and the chosen architecture 
can affect not only the performance and stability of the transistors but also the associated manufacturing costs. However, all OTFTs operate by the same working principle. An organic semiconductor thin-film, an insulator or dielectric material and three electrodes (source, drain and gate) comprise the basic layered structure of an OTFT. Before a bias is applied to the device, the organic semiconductor is unperturbed and a low concentration of free charge carriers are distributed evenly throughout the thin film (see Figure 2a). When a bias is applied to the gate electrode, an electric field is created and this causes the formation of an accumulation layer at the semiconductor-dielectric interface (Figure 2b). Subsequent application of increasing bias to the drain electrode causes the charges to travel laterally along the channel between the source and the drain electrodes, initially in a linear regime; according to Ohm's law, V = IR(Figure 2c). When the drain voltage exceeds the magnitude of the gate voltage, charges are no longer able to accumulate at the semiconductor-dielectric interface, resulting in a 'pinch-off' region and the current saturates (saturation regime) with increasing sourcedrain voltage (Figure $2 \mathrm{~d}$ ).
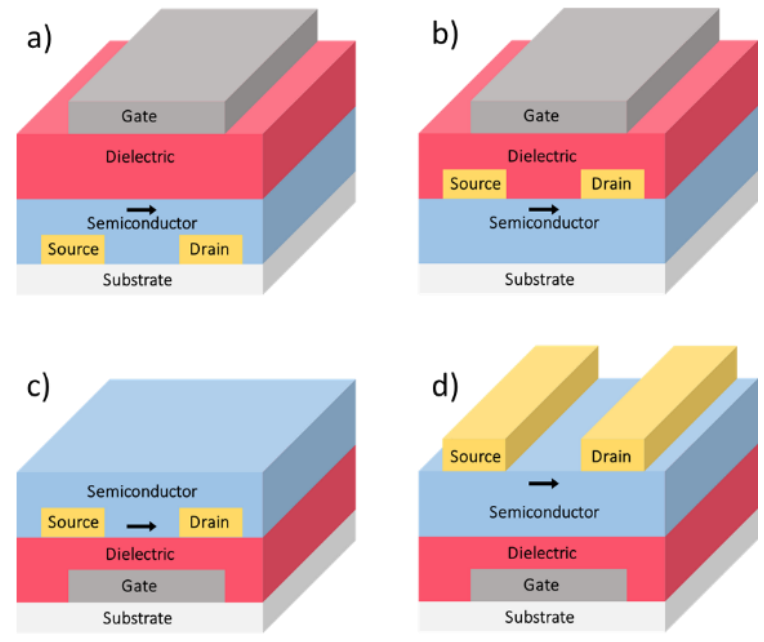

Figure 1. The four common OTFT device architectures, a) top-gate bottom-contact, b) top-gate top-contact, c) bottom-gate bottom-contact, d) bottom-gate top-contact. The arrows denote the flow of charge carriers in the device.

There are two significant pathways for charges to be transported between the source and drain electrodes in organic semiconducting polymer OTFTs; along the conjugated backbones of polymer chains or via hopping between chains. It should be noted that charge hopping occurs predominantly at the location of close $\pi-\pi$ interactions, as there is significant overlap of the highest occupied and lowest unoccupied molecular orbitals (HOMO and LUMO respectively). The HOMO and LUMO energies will be referred to herein as the ionization potential (IP) and electron affinity (EA) respectively. Solubilizing alkyl chains can play a detrimental role on charge hopping as they can sterically suppress close backbone contacts. With this in mind, the relative degree of order in the packing of polymer chains is often directly related to the OTFT mobility exhibited by a polymer. ${ }^{14,15}$ In highly ordered systems, the polymer chains often exhibit relatively short $\pi-\pi$ stacking distances, and the conjugated backbones are relatively rigid. This ensures that charge hopping, and transport along the backbone, are more efficient processes. Moreover, highly ordered polymer systems display a lesser degree of energetic disorder, reducing the likelihood of trap states within the thin film. ${ }^{16,17}$ Additionally, since OTFTs make use of the transport of charges in the plane of the channel, the in-plane mobility can be considered as the key factor in determining the performance of a device. As such, the orientation of the polymer packing can also play a key role in determining the charge carrier mobility in OTFTs. If a polymer exhibits an edge-on packing orientation, where the $\pi-\pi$ stacking occurs perpendicular to the plane of the OTFT substrate, the charge hopping occurs predominantly in the horizontal direction. This is the same direction as the charges are required to move through the transistor, and contributes towards the OTFT mobility. An example of this is the lamellar microstructure of poly(2,5-bis(thiophen-2yl)thieno[3,2-b]thiophene) (pBTTT), where the polymer backbones assemble in $\pi$-stacked lamella, with the backbones oriented out of plane with respect to the substrate, while the lamella are in plane. ${ }^{18,19}$ If a polymer exhibits a face-on packing orientation, where the $\pi-\pi$ stacking occurs parallel to the plane of the OTFT substrate, the charge hopping occurs predominantly in the vertical direction, which does not contribute significantly to the lateral charge carrier mobilities in OTFTs. 

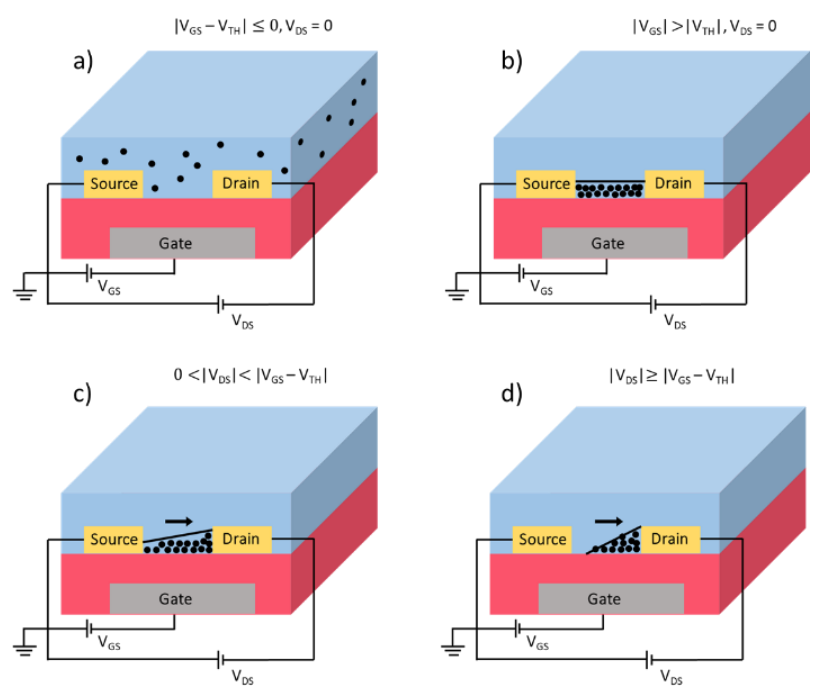

Figure 2. The basic working principles of a ptype bottom-gate bottom-contact OTFT device. $\mathrm{V}_{\mathrm{GS}}=$ gate voltage, $\mathrm{V}_{\mathrm{TH}}=$ threshold voltage and $\mathrm{V}_{\mathrm{DS}}=$ drain voltage.

The understanding of the relationship between semiconductor structure and OTFT performance has progressed significantly as the field has matured. Initially, it was generally accepted that a high degree of long-range order was the basic requirement to achieve high charge carrier mobilities. In particular, highly ordered edge-on oriented organic semiconductors were thought to be favorable, as a result of their excellent in-plane transport properties. ${ }^{20}$ Both the hopping between polymer chains, and intrachain transport along the backbone are strongly influenced by the order that the semiconductor is able to adopt, examples of highly ordered edge-on polymers include poly(3-hexylthiophene) (P3HT) and pBTTT. ${ }^{21,22}$ In later work, donor-acceptor (D-A) copolymers, such as indaceno[1,2-b:5,6-b']dithiophene-co2,1,3-benzothiadiazole (IDT-BT) and a thiopheneflanked diketopyrrolopyrrole copolymerized with thieno[3,2-b]thiophene (DPP-TT), were also reported to exhibit high mobilities in OTFT devices. $^{23,24}$ Surprisingly, some D-A copolymers often showed a distinct lack of long-range order, as indicated by $\mathrm{x}$-ray diffraction (XRD) measurements. These materials exhibit so called "short contacts", with no extensive $\pi$-stacking order. The polymer backbones can however adopt a highly planar conformation, facilitated by non-covalent interactions that act to minimize dihedral angles between repeat units, leading to low conformational disorder. Additionally, many of these materials adopt face-on orientations, which are associated with poor in-plane transport, and in some cases appear to be completely amorphous. This suggested that charge transport was predominantly along the conjugated polymer chains in such systems with occasional hopping between close contact interchain interactions. Often these polymers exhibited a donor-acceptor sequencing of the repeat units.

Indaceno[1,2-b:5,6-b']dithiophene (IDT) based copolymers with 2,1,3-benzothiadiazole (BT) are a prominent example of materials that display short-contact interactions, and high charge carrier mobility. ${ }^{23,25}$ Several molecular structural features contribute to the high charge carrier mobility exhibited by these polymers; two symmetric bridging $\mathrm{sp}^{3}$ carbon atoms help to rigidify the linked aromatic groups along the backbone, enhancing planarity and reducing energetic disorder, these bridgehead carbons are alkylated to confer solubility and contribute to interchain interactions. The presence of short-contact attraction between the IDT sulfur and BT nitrogen atoms further acts to planarise the backbone, which exhibits a relatively high torsional potential energy barrier for rotation. This highly planar conformation has an unusually low degree of energetic disorder as a result, despite the overall apparent low degree of crystallinity that has been observed for the polymers employing the IDT unit. ${ }^{26}$

Since it was first reported, the copolymer IDT-BT, which includes BT as the acceptor comonomer, has garnered significant interest, owing to the high hole mobility of up to $3.6 \mathrm{~cm}^{2} \mathrm{~V}^{-1} \mathrm{~s}^{-1}$ it was able to achieve in the saturation regime, and 2.0 $\mathrm{cm}^{2} \mathrm{~V}^{-1} \mathrm{~s}^{-1}$ in the linear regime. ${ }^{23}$ Initially, however, very few examples of chemical modifications to this polymer were reported, as a result of its relatively complex synthesis.

Herein, we summarize a number of design strategies used to modify the IDT-BT polymer that elucidate the relationship between the polymer structure and OTFT performance, contributing towards the improved rational design of new motifs for high-performance OTFT polymers.

In general, there are four key modifications that can be made to the structure of IDT-BT: (i) variation of the pendant alkyl chains from n-hexadecyl chains to shorter or branched chains; (ii) replacement the $\mathrm{sp}^{3}$ bridging carbon to the heavier atoms such as silicon and germanium or the use of an $\mathrm{sp}^{2}$ hybridized nitrogen atom; (iii) modification of the BT unit and (iv) extending the $\pi$-conjugated backbone of the IDT donor unit, such as the use of thiophenebenzo[b]indacenodithiophene (TBIDT) and dithiopheneindenofluorene (TIF) moieties. These modifications are summarized in Figure 3.

In this perspective we collate our previous studies that address each of these modifications. This demonstrates the fine-tuning of the structural or 
optoelectronic properties exhibited by the IDTbased polymers. It should be noted that comparison of the OTFT performance of materials published by different research groups can be misleading, since there are a number of factors that affect the mobility values reported such as the purity of the material, polymer molecular weight, the fabrication processes, device architecture, and calculation method. ${ }^{2}$ With this in mind, comparisons made herein are based upon OTFT devices using the same architecture and fabricated in the same laboratory, where possible.

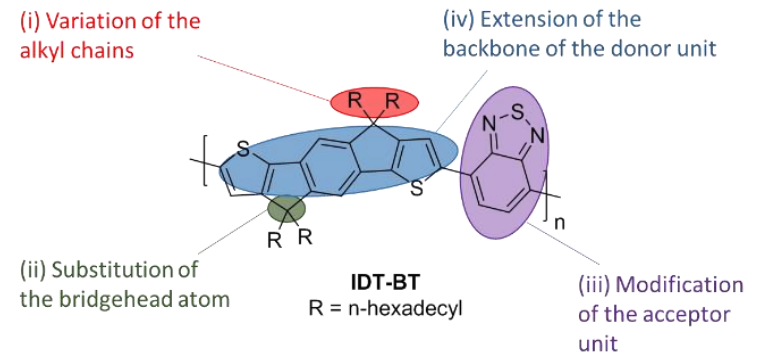

Figure 3. Basic design strategies for modification to the structure of IDT-BT.

\section{ALKYL CHAIN VARIATION}

A common, and synthetically straightforward, strategy that is often used to modulate the packing and order in polymers is the optimization of the solubilizing alkyl chains appended to the conjugated backbone. Not only do they ensure that the highly conjugated aromatic systems can be dissolved in common organic solvents, which is necessary for solution processing (one of the main benefits of OTFTs in comparison to their inorganic counterparts), but they can be used to control the aggregation and self-assembly properties of these polymeric semiconductors. Since the charge carrier mobility of organic semiconducting materials is related to the degree of close contacts that molecules, or polymer chains exhibit, in addition to a low energetic disorder, it follows that designing IDT-BT analogues which possess these properties should lead to the highest mobility materials.

This strategy has been shown previously in the optimization of polymers used in OTFTs, both using variations in alkyl chain length, and side chain branching points to control their structural and packing properties. ${ }^{27,28}$ In some cases, such as in Table 1. Summary of the properties of $\mathrm{C}_{1} \mathrm{C}_{4}$-IDT-BT, $\mathrm{C}_{2} \mathrm{C}_{6}$-IDT-BT, $\mathrm{C}_{8}$-IDT-BT and $\mathrm{C}_{16}$-IDT-BT.

\begin{tabular}{|c|c|c|c|c|c|c|c|}
\hline Polymer & $\begin{array}{c}\text { Mn/Mw } \\
(\mathrm{kDa})\end{array}$ & PDI & IP (eV) & EA (eV) & Eg (eV) & $\begin{array}{c}\text { Thin-film } \\
\lambda_{\max }(\mathrm{nm})\end{array}$ & $\begin{array}{c}\text { Average mobil- } \\
\text { ity }\end{array}$ \\
\hline $\begin{array}{c}\mathrm{C}_{1} \mathrm{C}_{4} \text {-IDT- } \\
\text { BT }\end{array}$ & $9 / 13$ & 1.4 & 5.3 & 3.6 & 1.7 & 655 & 0.003 \\
\hline
\end{tabular}

pBTTT, long linear alkyl units are able to interdigitate between neighboring chains, providing registration between out of plane lamella, and resulting in three dimensional ordering over a micron lengthscale. Moreover, in the case of an isoindigoco-dithiophene (IIDT) polymer, the OTFT hole mobility was enhanced by moving the side chain branching point further from the conjugated polymer backbone, relative to the parent polymer. ${ }^{28}$ As the branching point was moved further from the polymer backbone, the steric hinderance to $\pi$ $\pi$ stacking was reduced, allowing the polymer backbones to pack more intimately. This was demonstrated by the reduced $\pi-\pi$ stacking distance measured by grazing incidence wide-angle $\mathrm{X}$-ray scattering (GIWAXS).

Since the alkyl chains do not possess $\pi$ electrons, they act as insulators to charge carriers, suppressing charge hopping between polymer chains. Hence, shorter chains are preferable in this context. However, as mentioned above, the solubility of the polymer must also be taken into account, limiting the minimum length of alkyl chains that can be attached whilst still ensuring that the polymer is soluble. In addition to the requirement for solution processing, the polymer molecular weight that arises from the polymerization process also impacts upon the solubility. Since charge carriers are transported along polymer backbones, it is therefore preferential to produce high molecular weight polymers. Moreover, the sterics of these alkyl chains impact the packing of the polymer chains, dictating the degree of order in this system. Generally, linear alkyl chains provide fewer unfavorable steric interactions than branched chains, and in some cases linear alkyl units of adjacent polymer chains can intercalate, which provides improved order, resulting in fewer traps, and closer $\pi-\pi$ stacking distances between chains. With this in mind, four distinct alkyl chains were selected to elucidate the alkyl chain effect upon charge carrier mobility for an IDT-BT polymer backbone: 2-methylbutyl, 2ethylhexyl, n-octyl and n-hexadecyl. ${ }^{29}$ These are referred to as $\mathrm{C}_{1} \mathrm{C}_{4}$-IDT-BT, $\mathrm{C}_{2} \mathrm{C}_{6}$-IDT-BT, $\mathrm{C}_{8}$-IDT$\mathrm{BT}$ and $\mathrm{C}_{16}$-IDT-BT respectively, and summarized in Figure 4. 


\begin{tabular}{|c|c|c|c|c|c|c|c|}
\hline $\begin{array}{c}\mathrm{C}_{2} \mathrm{C}_{6} \text {-IDT- } \\
\text { BT }\end{array}$ & $40 / 87$ & 2.2 & 5.3 & 3.6 & 1.7 & 660 & 0.57 \\
\hline C8-IDT-BT & $33 / 243$ & 7.4 & 5.3 & 3.7 & 1.6 & 674 & 0.15 \\
\hline C $_{16}$-IDT-BT & $38 / 108$ & 2.8 & 5.4 & 3.7 & 1.7 & 677 & 1.2 \\
\hline
\end{tabular}

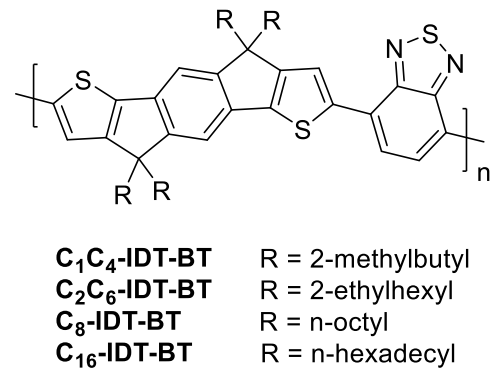

Figure 4. The structures of $\mathrm{C}_{1} \mathrm{C}_{4^{-}}, \mathrm{C}_{2} \mathrm{C}_{6^{-}}, \mathrm{C}_{8^{-}}$and $\mathrm{C}_{16}$-IDT-BT.

Each of these polymers were synthesized using the same methodology. The IDT monomers were synthesized by a Friedel-Crafts cyclization of 2,5dithien-2-yltherephthalic acid to form the indacenodithiophene-4,9-dione, followed by a WolffKishner reduction, alkylation and bromination. These IDT monomers were then copolymerized with the bis-pinacol ester of BT in a Suzuki crosscoupling reaction, to produce a series of polymers, as displayed in Scheme $1 .{ }^{25,29}$ As the varying alkyl chains impart different solubility upon the IDT monomers, and their resultant IDT-BT polymer chains, it was not straightforward to ensure that the polymer properties were consistent, as seen by the varying number average molecular weight $\left(\mathrm{M}_{\mathrm{n}}\right)$ and polydispersity index (PDI) of the polymers, shown in Table 1. Despite this, it is still possible to draw meaningful conclusions from the properties and performance of these various IDTBT polymers.

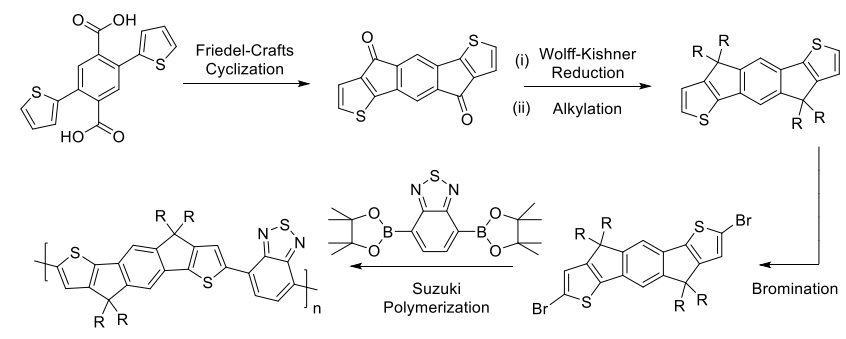

Scheme 1. Synthetic procedure of IDT-BT.

Top-gate bottom-contact OTFT devices were fabricated using each of the polymers, in order to evaluate the effect of the alkyl chains upon the hole mobility. From Table 1 it can be seen that $\mathrm{C}_{16^{-}}$ IDT-BT possesses the greatest hole mobility (1.2 $\left.\mathrm{cm}^{2} \mathrm{~V}^{-1} \mathrm{~s}^{-1}\right){ }^{29}$ Surprisingly, the branched chain $\mathrm{C}_{2} \mathrm{C}_{6}$-IDT-BT was able to outperform the straight chain $\mathrm{C}_{8}$-IDT-BT. The greater steric bulk of the branched chains is likely to hinder packing, in comparison to its straight chain analogue, however, this is not reflected by the hole mobilities obtained. The extremely high molecular weight and PDI of the $\mathrm{C}_{8}$-IDT-BT made processing uniform films of this polymer very difficult; poor film quality may contribute to the lower mobility exhibited by $\mathrm{C}_{8}$-IDT-BT. Nonetheless, the hole mobility of the $\mathrm{C}_{2} \mathrm{C}_{6}$-IDT-BT $\left(0.57 \mathrm{~cm}^{2} \mathrm{~V}^{-1} \mathrm{~s}^{-1}\right)$ is approximately only half the value of the best performing $\mathrm{C}_{16}$-IDT-BT, despite the greater degree of disorder likely to be present as a result of its branched side chains. ${ }^{29}$ This would suggest that in IDT-BT, the highly planar and low torsion backbone (ribbonlike) is likely to dominate the structural properties and the forming of lamellar stacks in the polymer, and the nature of the pendant alkyl chains have a limited effect on tuning these properties. The extremely low hole mobility of the $\mathrm{C}_{1} \mathrm{C}_{4}$-IDT$\mathrm{BT}$ is likely to be a result of the much lower molecular weight of this polymer. This is likely a result of the poorer solubility of the $\mathrm{C}_{1} \mathrm{C}_{4}$-IDT monomer, resulting in the precipitation of polymer chains during the polymerization.

From the thin-film UV-visible spectra it is clear that the linear chain IDT-BT polymers are more strongly aggregated than their branched chain counterparts, this can be seen by the red-shift in the absorption maximum wavelength $\left(\lambda_{\max }\right)$ in thin-films for the $\mathrm{C}_{8^{-}}$and $\mathrm{C}_{16}$-IDT-BT. ${ }^{29}$ This is a result of a slight narrowing of the bandgap, manifested in a $0.1 \mathrm{eV}$ increase in the electron affinity. Narrowing of the bandgap is indicative of greater aggregation and structural order in the linear side-chain polymers. Another feature that is present in the UV-vis spectrum of the $\mathrm{C}_{16}$-IDT-BT, is a pronounced shoulder-peak at approximately 575 $\mathrm{nm}$. This shoulder is much less pronounced in the cases of the other polymers in the series, which would suggest a greater degree of order in the solid-state aggregation of $\mathrm{C}_{16}$-IDT-BT. This correlates well with the highest hole mobility measured for this polymer. 
It is clear that the hole mobility of IDT-BT polymers is significantly influenced by the molecular weight of the polymer. As discussed above, one of the main transport pathways for charge carriers is along the polymer chains, thus longer chains require less hopping, and more efficient charge transport, an observation noted in similar studies. ${ }^{30}$ The length of the alkyl chains can influence the molecular weight that can be obtained via the polymerization, as shorter chains will render the IDT monomer, and subsequent polymer chains, less soluble. Also, it is clear that the greater aggregation and ordered packing exhibited by the $\mathrm{C}_{16}$-IDT-BT led to the best hole mobility in this series of polymers. Thus, relatively long, linear sidechains should be used to obtain high mobility IDTBT polymers. This affords; (i) sufficient solubility of the polymer, such that reasonable molecular weights can be achieved and (ii) a greater aggregation tendency, increasing the short contacts between polymer chains.

\section{BRIDGING ATOM SUBSTITUTION}

Another tactic that allows control over the structural and optoelectronic properties of conjugated units, consisting of multiple bridged aromatic rings, is the variation of the bridgehead atoms. In the cases where the bridgehead atom is a Group 14 element, such as carbon, silicon or germanium, it is not part of the $\pi$-conjugated system, as it is $\mathrm{sp}^{3}$ hybridized. As such, these bridgehead substitutions are used to tune the structural properties of the polymers in particular. Conversely, if the carbon bridgehead atoms are replaced with a Group 15 atom, such as nitrogen, the bridgehead becomes $\mathrm{sp}^{2}$ hybridized, and therefore part of the $\pi$-conjugated system. This allows tuning of both structural and optoelectronic properties

The larger atomic radii of silicon and germanium, in comparison to carbon, afford longer bond lengths between the bridgehead atom and the first carbon of the alkyl chain. Therefore, the alkyl chains are pushed further away from the conjugated polymer backbone, allowing greater $\pi-\pi$ interactions between polymer chains. This allows greater interaction between neighboring polymer backbones, leading to an increase in the crystallinity of such polymers. ${ }^{31-34}$ Moreover, the larger atomic radii of silicon and germanium result in lengthening of the bonds between the bridgehead atom and the adjacent carbon atoms of the fused conjugated system..$^{32}$ As the aromatic rings move further apart, the intramolecular transfer integral decreases and the IP decreases, as it has antibonding character with a node between the rings.
The antibonding lobes of this bond are pushed further from one another with increasing atomic radius, which results in a deepening of the IP. ${ }^{32,35}$ Another effect that the substitution of the carbon atom for a silicon atom, at the bridgehead, can have on the optoelectronic properties is a reduction in the EA. Specifically in the case of silicon, its $\sigma^{*}$ orbital can undergo effective mixing with the butadiene fragments of the adjacent thienyl or phenyl units. This stabilizes the LUMO, leading to a reduction in bandgap in the case of some $\mathrm{Si}$ bridged fused ring systems, relative to the carbonbridged analogue. ${ }^{35}$ Since the nitrogen bridging atom in NIDT is $\mathrm{sp}^{2}$ hybridized, the alkyl chains are directed outwards, in the plane of the polymer backbone. This leads to greater planarity and improved $\pi-\pi$ interactions between polymer chains. Another key effect of the substitution of a nitrogen atom at the bridgehead, is that it becomes part of the conjugated system. The nitrogen atom is relatively electron-donating, which results in a stabilization of the IP.

The silicon, germanium and nitrogen bridged analogues of IDT-BT have all been synthesized, and will be referred to as SiIDT-BT, GeIDT-BT and NIDT-BT respectively. ${ }^{36-38}$ It should be noted that these polymers were synthesized with different pendant alkyl chains, this is summarized in Figure 5. As discussed above, the choice of alkyl chains can also have an influence on the structural properties, and subsequently the charge carrier mobility of the IDT-BT polymers. Therefore, these polymers have been compared to a parent IDT-BT polymer with the same solubilizing chains, where possible. The $\mathrm{sp}^{2}$ hybridization of the bridgehead in NIDT-BT allows the possibility of only one pendant alkyl chain at the bridgehead, therefore a branched 2-octyldodecyl group is used. This is relatively similar to the two n-octyl chains situated on the carbon bridgehead in $\mathrm{C}_{8}$-IDT-BT, which will therefore be used as the comparison to NIDT-BT.

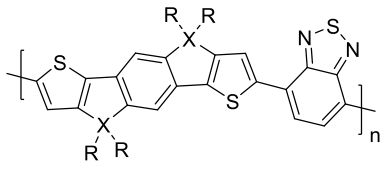

SilDT-BT $\quad X=S i \quad R=$ n-octyl GeIDT-BT $\quad X=G$ e $R=$ 2-ethylhexyl

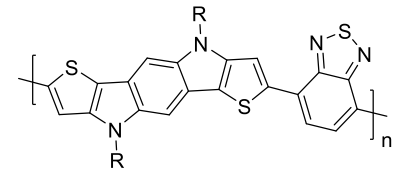

NIDT-BT R = 2-octyldodecyl
Figure 5. The structures of SiIDT-BT, GeIDT-BT and NIDT-BT.

The SiIDT and GeIDT monomers were both synthesized in a similar manner. ${ }^{36,37}$ First, a trimethylsilane (TMS) protected 2,2'-(2,5-dibromo-1,4phenylene)bis(3-bromothiophene) was lithiated, before addition of either a dichlorodialkylsilane 
or a dichlorodialkylgermane, leading to ring closure around the respective silicon or germanium bridgeheads. The SiIDT and GeIDT units were then brominated. It should be noted that the same polymerization conditions could not be used in the syntheses of SiIDT-BT and GeIDT-BT, owing to the poor stability of the SiIDT unit to basic conditions, such as those used in Suzuki cross-coupling reactions. Hence, in the case of the silicon bridge analogue, Stille cross-coupling reactions were used to polymerize SiIDT with the BT comonomer. In the synthesis of the NIDT unit, a Buchwald-Hartwig cross-coupling reaction was used to ring close 2,2'-(2,5-dibromo-1,4-phenylene)bis(3-bromothiophene) around two alkylamines. Lithiation then allows the formation of the bis-trimethylstannane NIDT monomer, which was subsequently copolymerized with the dibromo BT comonomer. ${ }^{38}$ The syntheses of SiIDT, GeIDT and NIDT units are summarized in Scheme 2.
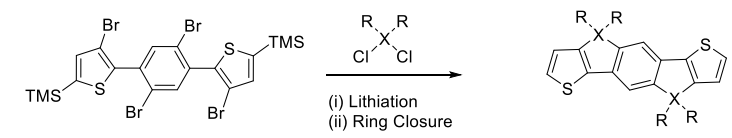

$\mathrm{X}=\mathrm{Si}$ or $\mathrm{Ge}$
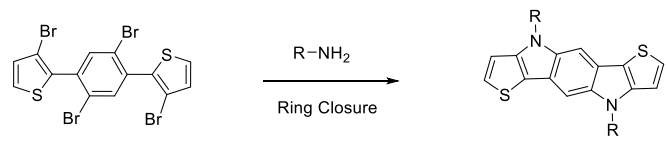

Scheme 2. Synthetic procedures for SiIDT-BT, GeIDT-BT and NIDT-BT.

By first considering the optoelectronic properties (summarized in Table 2) of SiIDT-BT, relative to $\mathrm{C}_{8}$-IDT-BT, it is clear that in this case the Si bridging atom has effectively widened the bandgap of the polymer to $1.8 \mathrm{eV}$. This is caused by a slight deepening of the HOMO, a result of the longer Si$\mathrm{C}$ bond separating the antibonding lobes of the conjugated thienyl and phenyl units, and a slight deepening of the IP. However, despite the predicted stabilizing interaction of the LUMO by the Si $\sigma^{*}$ orbital with the butadiene fragments of the neighboring thienyl and phenyl units, the EA is in fact shallower than measured for IDT-BT. It should be noted that this difference in EA to IDT$\mathrm{BT}$ is within the range of experimental error, and differences in the polymer molecular weights of SiIDT-BT and $\mathrm{C}_{8}$-IDT-BT

Table 2. Summary of the properties of SiIDT-BT, GeIDT-BT and NIDT-BT.

\begin{tabular}{|c|c|c|c|c|c|c|c|}
\hline Polymer & $\begin{array}{c}\mathrm{Mn} / \mathrm{Mw} \\
(\mathrm{kDa})\end{array}$ & PDI & IP (eV) & EA (eV) & Eg (eV) & $\begin{array}{c}\text { Thin-film } \\
\lambda_{\max }(\mathrm{nm})\end{array}$ & $\begin{array}{c}\text { Average mobil- } \\
\text { ity }\left(\mathrm{cm}^{2} \mathrm{~V}^{-1} \mathrm{~s}^{-1}\right)\end{array}$ \\
\hline SiIDT-BT & $30 / 56$ & 1.9 & 5.4 & 3.6 & 1.8 & 634 & $0.008^{36}$ \\
\hline GeIDT-BT & $32 / 73$ & 2.3 & 5.2 & 3.5 & 1.7 & 644 & $0.005^{37}$ \\
\hline NIDT-BT & $7 / 10$ & 1.4 & 4.9 & 3.4 & 1.5 & 770,830 & $0.030^{38}$ \\
\hline
\end{tabular}

may also be responsible for this unexpected observation. This wider bandgap is reflected in a hypsochromic shift in the thin-film absorption spectrum of SiIDT-BT. Additionally, the shoulder at $590 \mathrm{~nm}$ is more pronounced than the corresponding shoulder in $\mathrm{C}_{8}$-IDT-BT. ${ }^{36}$ In the case of GeIDT-BT, the bandgap (1.7 eV), IP and EA values are comparable to the corresponding $\mathrm{C}_{2} \mathrm{C}_{6}$-IDT-BT, indicating that the use of the Ge bridging atom has a minimal effect on the polymer's frontier molecular orbital energies. The thin-film absorption spectrum of GeIDT-BT exhibits a slight (15 nm) hypsochromic shift in both the absorption onset and $\lambda_{\max }$, suggesting that the solid-state bandgap of GeIDT-BT is marginally wider than that of $C_{2} C_{6}$ -
IDT-BT. Interestingly, the shoulder at approximately $600 \mathrm{~nm}$ is much more pronounced in GeIDT-BT, which implies that the Ge bridged polymer has greater order in its solid-state packing than the carbon bridged analogue, likely due to the alkyl chains protruding further from the backbone, as discussed earlier. ${ }^{37}$ As one might expect, the optoelectronic properties of NIDT-BT exhibit the greatest deviation from those of IDT-BT. The inclusion of the electron donating nitrogen into the conjugated system leads to a significantly shallower IP, and also a slight raising of the EA. This results in the much narrower bandgap exhibited in NIDT-BT $(1.5 \mathrm{eV})$. The greater electron do- 
nating character of the NIDT unit is likely to impart greater push-pull hybridization on the copolymer, leading to a reduction in the optical bandgap. Another contributing factor to the narrower bandgap of NIDT-BT may be improved backbone planarity, and consequently more ordered short-range aggregation of polymer chains. The thin-film UV-vis spectrum of NIDT-BT shows a very pronounced shoulder, which is larger in magnitude than the $S_{o}-S_{1}$ peak. This is indicative of strong aggregation of the polymer chains, likely arising from the planarity of the bridgehead, and the first carbons of the pendant alkyl chain, which extend in the plane of the conjugated backbone. ${ }^{38}$

Further investigation into the structural properties were also undertaken for SiIDT-BT and GeIDT-BT to elucidate the effect of bridgehead substitution upon the hole mobility of the polymers. For the analogous branched chain $\left(\mathrm{C}_{2} \mathrm{C}_{6}-\right) \mathrm{Si}-$ IDT-BT, differential scanning calorimetry (DSC) analysis revealed the presence of a small melting transition at $260^{\circ} \mathrm{C}$, whilst no thermal transitions were present in the analogous $\mathrm{C}_{2} \mathrm{C}_{6}$-IDT-BT, indicating that the inclusion of the Si bridgehead atoms has led to an increase in crystallinity. Moreover, the presence of a much sharper melt at 290 ${ }^{\circ} \mathrm{C}$, for GeIDT-BT, suggests a greater degree of order and aggregation in this polymer, relative to both IDT-BT and SiIDT-BT. ${ }^{39}$ GIWAXS was performed on thin-films of GeIDT-BT to further probe its polymer packing; revealing that it was able to form semi-crystalline films, whilst the analogous $\mathrm{C}_{2} \mathrm{C}_{6}$-IDT-BT polymer was amorphous, by comparison. ${ }^{37}$ The presence of (100) and (200) reflections in the out-of-plane direction are consistent with the formation of polymer lamellae, with dspacings of 14.0 Å. Additionally, (010) reflections in the in-plane direction revealed that the $\pi-\pi$ stacking distance was $3.9 \AA$ A. One other notable feature of GeIDT-BT's diffractogram was the presence of arcing in the (100) and (200) out-of-plane reflections, this suggests that the lamellar stacks are not well aligned with respect to the surface of the substrate.

The hole mobility of SiIDT-BT, GeIDT-BT and NIDT-BT were extracted from the electrical characteristics of OTFT devices, summarized in Table 2. In the cases of the Si- and Ge-bridged IDT polymers, top-gate bottom-contact OTFTs were prepared, however bottom-gate top-contact OTFT devices were fabricated for NIDT-BT. This difference in device architecture is known to affect the charge carrier mobilities, and so this must also be taken into account when drawing conclusions from the results of the NIDT-BT based devices. Despite the improvement in aggregation and $\pi-\pi$ stacking of polymer chains imparted by the use of larger Group 14 atoms, the measured hole mobilities are significantly worse $\left(<0.01 \mathrm{~cm}^{2} \mathrm{~V}^{-1} \mathrm{~s}^{-1}\right)$ than their carbon-bridged analogues..$^{36,37}$ It has been suggested that the mobility may be limited by grain boundaries and misaligned lamellae, caused by the greater degree of aggregation in these systems. By comparison, the hole mobility of NIDT-BT $\left(0.03 \mathrm{~cm}^{2} \mathrm{~V}^{-1} \mathrm{~s}^{-1}\right)$ was higher than the values seen in either SiIDT-BT or GeIDT-BT. ${ }^{38}$ This may be, in part, due to the difference in device architecture used, but may also be due to enhanced backbone planarity and aggregation. Despite this, the low $\mathrm{M}_{\mathrm{n}}$ obtained for NIDT-BT, which is likely an effect of the poor solubility of the more planar and aggregating polymer chains, may significantly limit the charge carrier mobility in this particular NIDT-BT batch. Hence, the hole mobility is still an order of magnitude lower than that measured for $\mathrm{C}_{8}$-IDT-BT, and it should also be noted that the $\mathrm{C}_{8}$-IDT-BT mobility is poorer than expected due to the high molecular weight of this polymer batch, and subsequent issues processing uniform thin-films. ${ }^{29}$

Overall, the use of bridging atom substitution does not appear to be an effective strategy to improve the hole mobility of IDT-BT polymers. Despite improving the degree of aggregation and order in the polymers, this did not translate to improved charge carrier mobilities in OTFTs. There are a number of possible reasons for this, including: grain boundary limitations, misalignment of polymer lamellae or the attainment of lower polymer molecular weights as a result of reduced solubility of polymer chains. There may be improvements in the hole mobilities that can be achieved with SiIDT-BT, GeIDT-BT and NIDT-BT through rigorous optimization of device processing, however it is unlikely that the values will match or surpass those achieved by the carbon-bridged analogues, which are currently 1-2 orders of magnitude larger.

\section{MODIFICATION OF THE BENZOTHIADIAZOLE COMONOMER}

Modification or substitution of the BT comonomer are amongst the simplest chemical alterations that can be made to IDT-BT. Whilst the small and rigid $\mathrm{BT}$ unit has been utilized to impart a high degree of backbone order in IDT-BT, variation of the comonomer can afford the ability to tune the polymer properties. Throughout literature on thiophene-based organic semiconductors, there are a number of strategies that have been commonly used to impart a high degree of order and planarity along polymer backbones; namely the use of 
attractive forces such as S---F intramolecular interactions, or the reduction of repulsive steric interactions, by replacing phenyl groups with thienyl groups to promote thienyl-thienyl (rather than thienyl-phenyl, or phenyl-phenyl) linkages, for example. ${ }^{40,41}$ These same tactics were adopted in the case of the IDT-BT polymer, whereby modified versions of the BT unit were used as the comonomer, these are summarized in Figure 6.

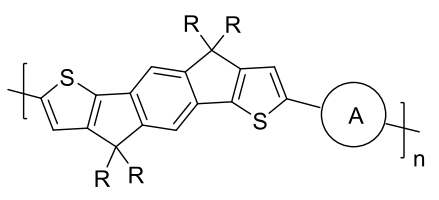

$A=$
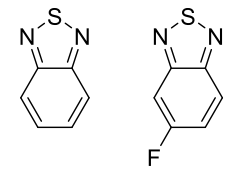

IDT-BT

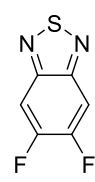

IDT-DFBT

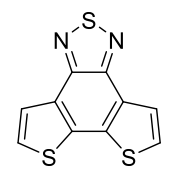

IDT-DTBT
Figure 6. The structures of IDT-BT, IDT-FBT, IDTDFBT and IDT-DTBT.

The inclusion of either one or two fluorine atoms on the BT monomer in the 5 and 6 positions (FBT and DFBT respectively) has been speculated to encourage backbone planarity owing to the strong electrostatic interactions between sulfur atoms of thienyl moieties with the fluorine atoms of the BT unit. ${ }^{2}$ However, top gate-bottom contact OTFT devices of IDT-FBT and IDT-DFBT exhibited lower hole mobilities than IDT-BT, where the $\mathrm{C}_{16}$-IDT monomer is used in each of the polymers. IDT-FBT and IDT-DFBT were only able to produce average hole mobilities of 0.9 and $0.5 \mathrm{~cm}^{2} \mathrm{~V}^{-1} \mathrm{~s}^{-1}$, in comparison to the average hole mobility of $1.2 \mathrm{~cm}^{2} \mathrm{~V}^{-1}$ $\mathrm{s}^{-1}$ measured for IDT-BT. ${ }^{29,43}$ In a similar study on SiIDT-BT, the BT comonomer was substituted for both FBT and DFBT. Specular XRD measurements indicated that the S---F interaction did indeed improve backbone planarity, manifesting in a greater degree of crystalline order in the films. Despite this, the FBT and DFBT containing polymers also exhibited lower hole mobilities. These results were rationalized by the deeper lying HOMO levels of the fluorinated analogues; this may induce contact resistance at the electrode interface. ${ }^{44}$

The addition of thienyl rings that have been fused to the periphery of the BT unit, to form DTBT, as shown in Figure 6, was expected to impart greater planarity along the polymer backbone by reducing the steric interactions between the C-C linkages when polymerized with IDT.43,45 Steric interactions between the hydrogen atoms alpha to an aryl-aryl linkage can cause torsion between the adjacent units, disrupting the planarity of the polymer backbone. As such, the inclusion of fused thiophenes on the periphery of the BT unit were adopted with a view to lessening these unfavourable steric interactions. Another consideration to be made is that the addition of the fused thienyl units forces the polymer backbone into a noncollinear arrangement. When top-gate bottom-contact OTFTs were fabricated using IDT-DTBT, however, the average hole mobility $\left(0.06 \mathrm{~cm}^{2} \mathrm{~V}^{-1} \mathrm{~s}^{-1}\right)$ was substantially lower than that of IDT-BT (1.2 $\left.\mathrm{cm}^{2} \mathrm{~V}^{-1} \mathrm{~s}^{-1}\right) .^{29,45}$ This significant decrease in hole mobility is indicative of major disruption to the order and planarity of the polymer backbone. This was verified using photothermal deflection spectroscopy (PDS). PDS measures the sub-bandgap absorption, which can be used in order to extract the Urbach energy $\left(E_{U}\right)$. The Urbach energy is related to structural and phonon state disorder in a material, and can therefore be used to compare the relative structural order between material systems. In the case of IDT-BT, the extracted $\mathrm{E}_{\mathrm{U}}$ was $24 \mathrm{meV}$, compared to the significantly larger $37 \mathrm{meV}$ extracted for IDT-DTBT. ${ }^{43}$ This suggests that the use of noncollinear units such as DTBT in fact induce conformational disorder in the polymer backbone, which leads to a significant decrease in hole mobility. Alternatively, this may be a result of reducing the stabilizing interactions between the peripheral thienyl units on IDT with the adjacent BT, this is discussed in more detail below.

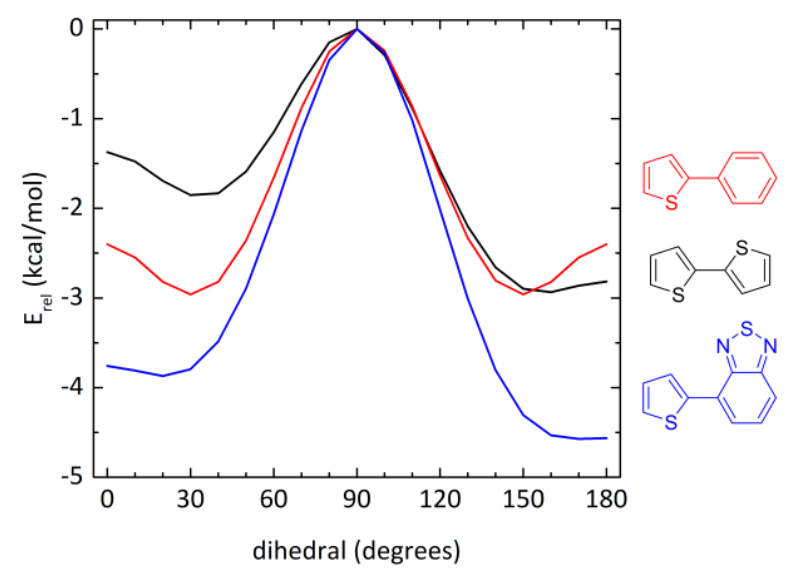

Figure 7. The computed torsional potential energy surfaces of thienyl-phenyl, thienyl-thienyl and thienyl-BT linkages.

The relative lack of success achieved with the above modifications to BT suggest that this unit is likely to be integral to the highly ordered and planar backbone in IDT-BT, which is so beneficial for charge transport. Density functional theory (DFT) 
calculations can be used to elucidate how the BT unit is well suited to preserve backbone planarity in IDT-BT. Computed torsional potential energy surfaces (PES) of a thienyl-BT (T-BT), thienylthienyl (T-T) and thienyl-phenyl (T-P) linkages are displayed in Figure 7. The T-P link has a symmetrical torsional PES, while a T-T is more stable in the anti-configuration (close to $180^{\circ}$ ) as the larger bond angles of the 5-membered rings relieve steric strain between the sulfur atom of one thiophene with the alpha proton of the other ring. However, $\mathrm{T}-\mathrm{T}$ is less stable in the syn configuration than T-P due to large exchange repulsion between sulfur atoms close in space. Therefore, the assumption in the design of IDT-DTBT to relieve steric strain is correct based only on the interactions of the alpha protons. The T-BT link is more stable than both T-T and T-P in both the anti and the syn conformations, despite the steric clashes of protons. This stabilization must arise from other more favorable non-covalent interactions. With this in mind, the tactic of reducing steric interactions around the linkage by the use of the fused dithienyl BT unit, may have acted to diminish the favorable through-space stabilizing interactions between the IDT and BT units. Hence, there would be a greater probability of torsion around the bond between monomer units, and less driving force for backbone order, which may limit the charge carrier mobility of IDT-DTBT. This is also supported by the comparatively low hole mobility of $0.15 \mathrm{~cm}^{2} \mathrm{~V}^{-1} \mathrm{~s}^{-1}$ measured in an IDT-based polymer where thieno[3,2-b]thiophene is used as the comonomer (IDT-TT). ${ }^{25}$

Figure 8 summarizes the various non-covalent interactions of the two different conformers associated with the IDT-BT link. The dominating interactions present in these systems were explored through the use of natural bond orbital (NBO) calculations. In conformer (1) there are stabilizing through-space resonance interactions between the lone pair of the $\mathrm{N}$ with the neighboring $\mathrm{S}-\mathrm{C}^{*}$ antibonding orbital and the lone pair of the $\mathrm{S}$ atom with the $\mathrm{N}-\mathrm{C}^{*}$ and $\mathrm{N}-\mathrm{S}^{*}$ antibonding orbitals. In general, the large repulsive exchange energies associated with these atoms being closer than the sum of their van der Waals radii are larger than the resonance stabilization, but lone pair donation certainly contributes to stabilizing more planar geometries. A larger contribution comes from electrostatic interactions. ${ }^{46}$ Despite the electron rich nature of sulfur, it has a partial positive charge in IDT, owing to the donation of one of its lone pairs into the conjugated unit. As such, there is a resultant electrostatic attraction to the negatively charged $\mathrm{N}$ of the BT unit. Therefore, despite the destabilizing steric interaction of the two hydrogen atoms on the alternate side of the IDT-BT linkage, and the sizeable steric repulsion between $\mathrm{S}$ and $\mathrm{N}$ atoms, this conformer is overall very stable. Conformer (2) is accessed upon $180^{\circ}$ rotation about the IDT-BT linkage. From the computed torsional PES, conformer (2) is preferred slightly over conformer (1), as a result of further stabilizing interactions present in this conformation. In this case, through-space resonance interactions are present on both sides of the IDT-BT linkage; the IDT sulfur's lone pair is able to interact with the neighboring $\mathrm{C}-\mathrm{H}^{*}$ antibonding orbital of $\mathrm{BT}$, and the nitrogen lone pair of $\mathrm{BT}$ is able to interact with the $\mathrm{C}-\mathrm{H}^{*}$ antibonding orbital of the IDT unit. There is electrostatic attraction between the $\mathrm{N}$ atom of BT with the neighboring hydrogen of IDT, in what can be considered as a non-traditional hydrogen bond. ${ }^{47}$ These combined attractive forces once again outweigh the associated steric exchange energies between atoms in such close proximity. The net result of these additional stabilizing interactions in the case of IDT-BT's preferred conformers is a larger barrier to rotation, relative to traditional $\mathrm{T}-\mathrm{T}$ and $\mathrm{T}-\mathrm{P}$ type linkages, as can be seen in Figure 7. As such, IDT-BT has a much greater driving force to adopt highly planar backbone conformations. The energetic barrier to rotation is so large that it is likely that both conformers (1) and (2) are present in the polymer chain, as both are statistically possible during the polymer synthesis, but locked into the conformation due to the high energetic barrier. This has been previously observed in molecular dynamics simulations of amorphous IDT-BT. ${ }^{23}$

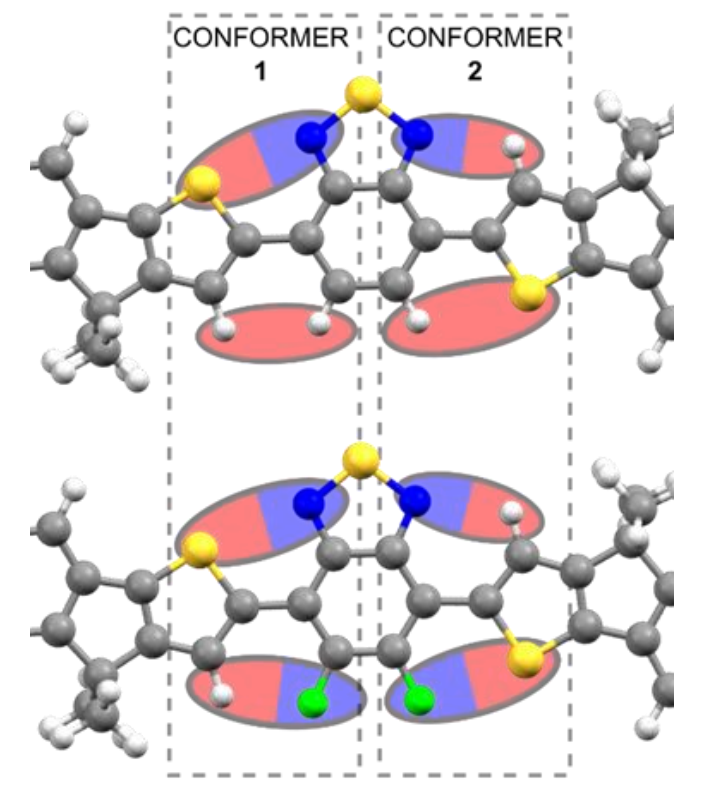

Figure 8. Non-covalent interactions in different conformations of IDT-BT (top) and IDT-DFBT 
(bottom). Red areas of the ovals represent a partial positive charge, while blue areas represent partial negative charge. Where grey atoms $=\mathrm{C}$, white atoms $=\mathrm{H}$, yellow atoms $=\mathrm{S}$, blue atoms $=$ $\mathrm{N}$, green atoms $=\mathrm{F}$.

The computed torsional PES of a thienyl-BT (T-BT) linkage was compared to that of thienyl-DFBT (TDFBT) to compare the energetic barriers to rotation in both IDT-BT and IDT-DFBT, shown in Figure 9. As one might expect, the introduction of the larger fluorine atoms increases the destabilizing steric repulsion in the conformers of T-DFBT, however this is offset by greater electrostatic attraction and the preservation of the stabilizing through-space resonance interactions between IDT and BT as discussed above. Comparison with a less planar thienyl-difluorophenyl system suggests that the $\mathrm{N}-\mathrm{H}$ interaction is more important than the S-F interaction. While IDT-BT links would result in one stabilizing and one destabilizing electrostatic interaction in each conformation, fluorination leads to stabilizing electrostatic interactions in both cases (Figure 8), particularly in the case of conformation (1) which is significantly stabilized over IDT-BT. These calculations suggest that the inclusion of the fluorine atoms on the BT unit in fact increase the energetic barrier to backbone torsion, which would promote backbone planarity. This was verified in the aforementioned study of a series of SiIDT containing polymers, in which specular XRD measurements verified a greater degree of crystalline order when FBT and DFBT were used as the comonomer, in comparison to BT. ${ }^{44}$ Despite this, the hole mobilities of IDTFBT and IDT-DFBT were measured to be lower than that of IDT-BT. Additionally, a comparative study on IDT-BT and IDT-DFBT, where thiophene spacers were included between the IDT and BT/DFBT units, also predicted greater planarity in the case of the fluorine containing polymer, but the hole mobility was an order of magnitude lower than the non-fluorinated BT analogue. ${ }^{48}$ Hence, it is clear that backbone planarity is not the only parameter that can influence OTFT hole mobility. There are several other factors which can also contribute to the charge transport properties. For example, the more planar backbone afforded by the S---F interactions in IDT-FBT and IDT-DFBT may render the polymer less soluble, restricting polymer molecular weight, as well as lead to an increase in solution aggregation, which in turn may affect film formation. It should be noted that whilst the IDT-FBT and IDT-DFBT hole mobilities are lower than that of IDT-BT, they are still an order of magnitude greater than IDT-DTBT, indicating that preservation of an IDT-BT linkage along the polymer backbone is advantageous for high hole mobility IDT-based polymers.

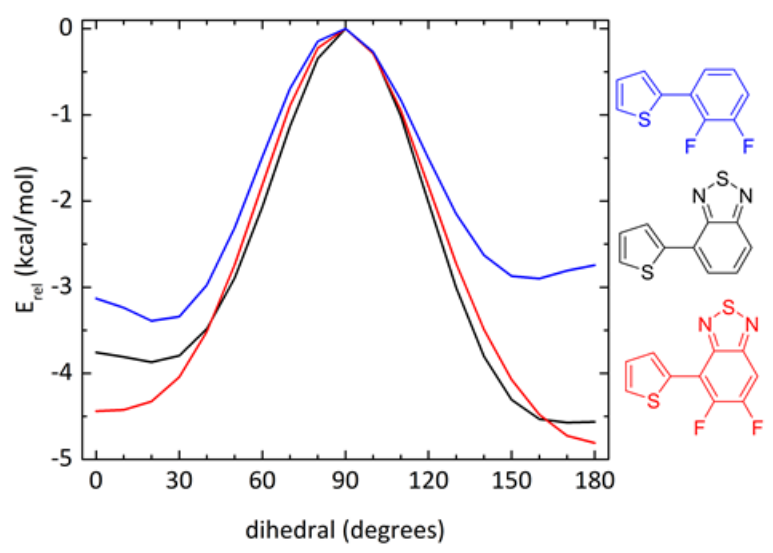

Figure 9. The computed torsional potential energy surfaces of thienyl-difluorophenyl, thienyl$\mathrm{BT}$ and thienyl-DFBT linkages.

\section{EXTENSION OF THE CONJUGATED IDT UNIT}

Another strategy that can be employed to modify the structure of IDT-BT is extending the $\pi$-conjugated system of the IDT unit with additional aromatic rings, such as phenyl or thienyl units. This was expected to increase the rigidity and coplanarity of the backbone, benefiting the intrachain transport through potentially reduced torsional disorder, and subsequently increase the hole mobilities that these polymers could achieve. Moreover, the extended $\pi$-conjugated units were expected to experience greater interchain $\pi-\pi$ overlap, allowing the additional possibility of improved interchain charge hopping. Three additional donor motifs, based on IDT, have been explored; indacenodithieno[3,2-b]thiophene (IDTT), thiophenebenzo[b]indacenodithiophene (TBIDT) and dithiopheneindenofluorene (TIF). These donor units were copolymerized with $\mathrm{BT}$ to form IDTT-BT, TBIDT-BT and TIF-BT respectively, the structures of which are summarized in Figure $10.49-51$
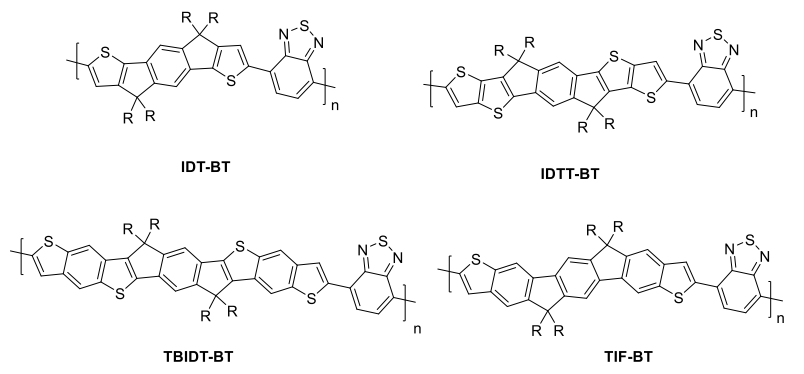

Figure 10. The structures of IDT-BT, IDTT-BT, TBIDT-BT and TIF-BT. 
The synthesis of the IDTT core can be considered as analogous to that of IDT, with the difference being the use of 2,5-Bis(thieno[3,2-b]thiophen-2yl)terephthalic acid as the starting reagent, before Friedel-Crafts cyclization, reduction, alkylation and bromination. ${ }^{25,49}$ The resultant monomer was copolymerized with bis-pinacol ester of BT using Suzuki polymerization conditions to yield IDTT-BT. In order to synthesize the TBIDT monomer, the $\mathrm{C}_{16}$-alkylated IDT monomer underwent formylation, coupling with a 3-bromo-2-hydroxymethyl-thiophene and oxidation yielded the key intermediate (A) shown in Scheme 3. Acidpromoted annulation was then used to ring close this intermediate to form TBIDT. This fused ring core was subsequently stannylated and reacted with a bis-bromo BT monomer under stille polymerization conditions, producing TBIDT-BT. TIF was synthesized via a Pd-catalyzed $\mathrm{C}-\mathrm{H}$ activation of the methyl groups of key intermediate (B) to form the ring closed product. The $\mathrm{sp}^{3}$ hybridized carbon bridgeheads were then alkylated with solubilizing $\left(\mathrm{C}_{16}\right)$ chains, and stannylated. Finally the TIF monomer was copolymerized with the dibrominated BT, in a Stille polymerization, resulting in the TIF-BT polymer. ${ }^{50}$ The molecular weights and PDIs of the IDTT-BT, TBIDT-BT and TIF-BT polymers are summarized in Table 3. In the cases of TBIDT-BT and TIF-BT these $\mathrm{M}_{n}$ and PDIs are comparable. However, the reference IDTBT and IDTT-BT polymer batches have a much greater molecular weights and larger polydispersity indices, which must be taken into account when comparing polymer properties.

Table 3. Summary of the properties of IDTT-BT, TBIDT-BT and TIF-BT.

\begin{tabular}{|c|c|c|c|c|c|c|c|}
\hline Polymer & $\begin{array}{c}\mathrm{Mn} / \mathrm{Mw} \\
(\mathrm{kDa})\end{array}$ & PDI & IP (eV) & EA (eV) & Eg (eV) & $\begin{array}{c}\text { Thin-film } \\
\lambda_{\max }(\mathrm{nm})\end{array}$ & $\begin{array}{c}\text { Average mobil- } \\
\text { ity }\left(\mathrm{cm}^{2} \mathrm{~V}^{-1} \mathrm{~s}^{-1}\right)\end{array}$ \\
\hline IDTT-BT & $76 / 196$ & 2.6 & 5.4 & 3.7 & 1.7 & 675 & 1.0 \\
\hline TBIDT-BT & $62 / 87$ & 1.4 & 5.5 & 3.6 & 1.9 & 595 & 0.90 \\
\hline TIF-BT & $57 / 83$ & 1.5 & 5.7 & 3.7 & 2.0 & 577 & 2.9 \\
\hline
\end{tabular}
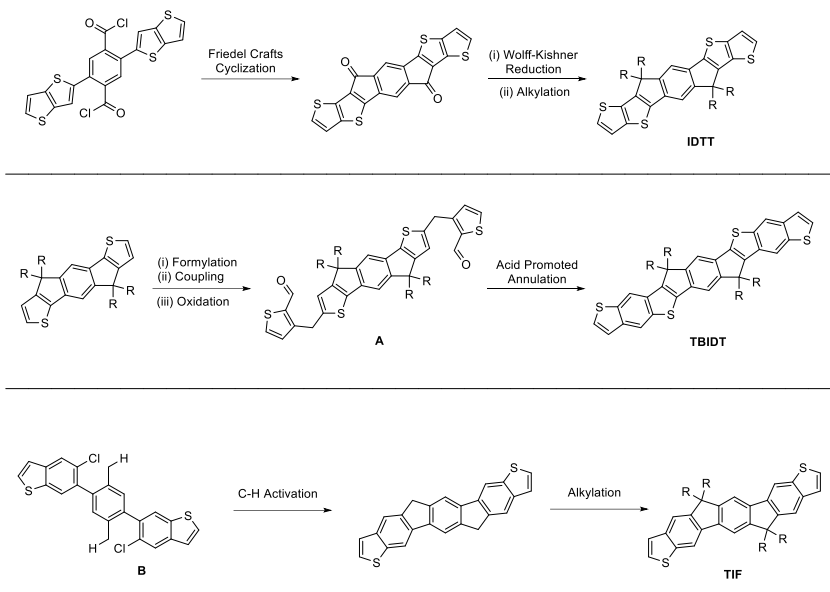

Scheme 3. Synthetic procedures for IDTT, TBIDT and TIF.

Top-gate bottom-contact OTFT devices were fabricated using each of IDTT-BT, TBIDT-BT and TIF$\mathrm{BT}$, the results of which are summarized in Table 4 and Figure 11. Thermal annealing was not utilized in the fabrication of any IDT-BT, IDTT-BT, TBIDT-BT or TIF-BT devices. The resultant average hole mobilities of IDTT-BT and TBIDT-BT devices (1.0 and $0.90 \mathrm{~cm}^{2} \mathrm{~V}^{-1} \mathrm{~s}^{-1}$ respectively) are marginally lower than that of IDT-BT. TIF-BT, on the other hand, was able to achieve a significantly greater average hole mobility than any of the previously discussed analogues of IDT-BT $\left(2.9 \mathrm{~cm}^{2} \mathrm{~V}\right.$ $\left.{ }^{1} \mathrm{~s}^{-1}\right) .{ }^{50}$ It should be noted that upon thermal annealing, OTFTs employing TIF-BT and TBIDT-BT exhibit degradation in their on-currents, which diminishes the OTFT performance. 


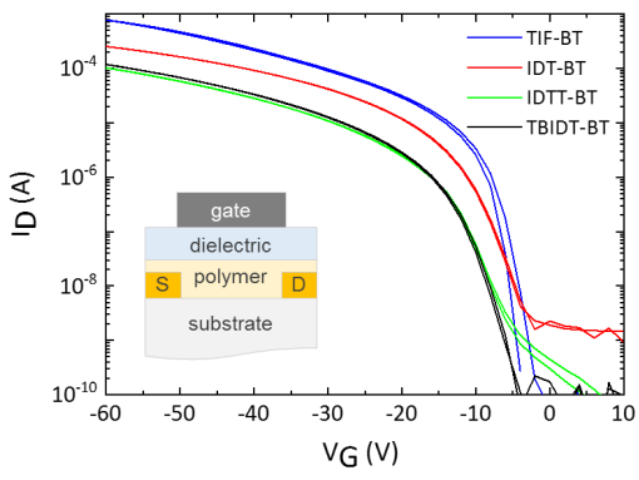

Figure 11. Saturation transfer characteristics of IDT-BT, IDTT-BT, TBIDT-BT and TIF-BT bottomgate top-contact OTFT devices.

Table 4 summarizes the optoelectronic properties of each of the extended core polymers. The IP and EA of IDTT-BT are very similar to IDT-BT, despite the presence of a higher backbone ratio of thiophene units. Both have an optical bandgap of 1.7 $\mathrm{eV}$, and a solid state $\lambda_{\max }$ of $\sim 670 \mathrm{~nm}$. It should be noted that IDTT-BT exhibits a less pronounced shoulder-peak (at $\sim 580 \mathrm{~nm}$ ) than that observed in IDT-BT. This would suggest that IDTT-BT exhibits less order in the solid state. ${ }^{49}$ TBIDT-BT, on the other hand, exhibited a slightly larger IP and a slightly smaller EA (both by $0.1 \mathrm{eV}$ ) than IDT-BT, resulting in a widening of the optical bandgap to $1.9 \mathrm{eV}$. This corresponds to a hypsochromic shift in $\lambda_{\max }$ to $570 \mathrm{~nm}$, with a shoulder at $550 \mathrm{~nm}$. Similarly, TIF-BT also exhibits a widening of the optical bandgap to $2.0 \mathrm{eV}$, in this case it is a manifestation of a greater IP $(5.7 \mathrm{eV})$, whilst maintaining a comparable EA to IDT-BT. This has been attributed to the lower ratio of the electron-rich thiophene units along the conjugated polymer backbone. The relatively wide bandgap of TIF-BT is also reflected in the thin-film UV-vis absorption spectrum, with a $\lambda_{\max }$ of $580 \mathrm{~nm}$ and a well-defined shoulder at $540 \mathrm{~nm}$, indicating a relatively large degree of order in the polymer thin-film. ${ }^{50}$

GIWAXS analysis has also been carried out on ascast and annealed thin-films (as per their OTFT fabrication) of IDT-BT, TBIDT-BT and TIF-BT, which can be seen in Supplementary Figures S1S3. In each of IDT-BT, TBIDT-BT and TIF-BT, there are a number of structural similarities. Each polymer displays relatively weak diffraction with a broad $\pi-\pi$ stacking peak in the out-of-plane $\left(\mathrm{q}_{\mathrm{z}}\right)$ direction. This is indicative of relatively weak semicrystalline order in each of these polymer thin-films. In IDT-BT, this diffraction peak is centered at $\mathrm{q}_{\mathrm{z}}=1.53 \AA^{-1}$, corresponding to a predominant $\pi-\pi$ stacking distance of $4.10 \AA$, whilst the predominant $\pi-\pi$ stacking distances in TBIDT-BT and TIF-BT are $4.22 \AA$ and $4.16 \AA$ respectively. This suggests that IDT-
BT possesses more favorable short contacts than either TBIDT-BT or TIF-BT, which one would expect to manifest in a greater charge carrier mobility. However, as noted above, TIF-BT in fact has a higher hole mobility than IDT$\mathrm{BT}$, indicating that short contacts are not the only prevalent feature necessary for high charge carrier mobility in ptype polymers. Interestingly, upon thermal annealing, the broad $\pi-\pi$ stacking peak of TIF-BT shifts from ${ }_{1.51} \AA^{-1}$ to $1.57 \AA^{-1}$, indicating a significant decrease in the $\pi$ $\pi$ stacking distance to $4.00 \AA$. Conversely, upon thermal annealing, IDT-BT and TBIDT-BT do not exhibit any significant decrease in their $\pi-\pi$ stacking distances. GIWAXS measurements had been previously carried out on as-cast and annealed IDTT-BT, revealing similar degree of semicrystalline order with a broad $\pi-\pi$ stacking peak at $\mathrm{q}_{\mathrm{z}}=1.55 \AA^{-1}$ in the as-cast sample. ${ }^{49}$ Upon thermal annealing, it was evident that the $\pi-\pi$ stacking peak at $\mathrm{q}_{\mathrm{z}}=1.55 \AA^{-1}$ became sharper, and another peak at $\mathrm{q}_{\mathrm{z}}=0.35 \AA^{-1}$ appeared. This would suggest that both face-on and edge-on domains exist within the annealed IDTT-BT thin-film.

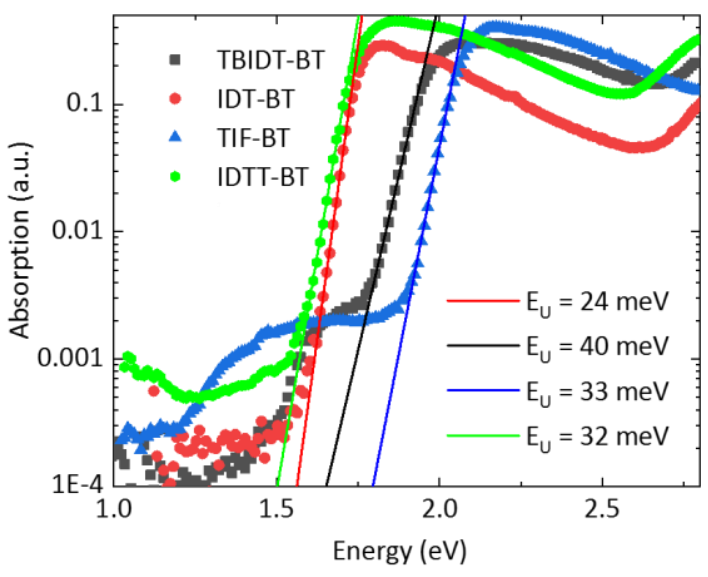

Figure 12. Absorption of IDT-BT, IDTT-BT, TBIDTBT and TIF-BT films, measured by photothermal deflection spec-troscopy. The solid line represents an exponential tail fit for extraction of the Urbach energies $E_{U}$.

To further elucidate differences in the structural order of these polymers, PDS analysis was also undertaken, the results of which are summarized in Figure 12. IDT-BT has one of the sharpest subbandgap absorptions recorded for a semiconducting polymer, corresponding to an Urbach energy of 24 meV. ${ }^{26}$ Notably, IDTT-BT, TBIDT-BT and TIFBT exhibit a higher degree of energetic disorder as compared to IDT-BT, upon inspection of their sub-bandgap absorptions, with Urbach energies of $32 \mathrm{meV}, 40 \mathrm{meV}$ and $33 \mathrm{meV}$, respectively (Figure 12)..$^{50}$ However, in the PDS spectra of TBIDT-BT and TIF-BT a sub-bandgap charge transfer absorp- 
tion feature is also observed, which can be associated to interchain charge transfer. ${ }^{51}$ This feature can be used to explain why TIF-BT is able to outperform IDT-BT despite its higher energetic disorder and relatively comparable microstructure. In the case of TBIDT-BT this feature may explain why, despite a relatively large Urbach energy (40 $\mathrm{meV}$ ), the polymer still exhibits charge carrier mobilities close to $1 \mathrm{~cm}^{2} \mathrm{~V}^{-1} \mathrm{~s}^{-1}$. It should be noted that IDT-based polymer systems with comparable energetic disorder have shown a far more pronounced drop in charge carrier mobility. ${ }^{43}$ This indicates that the improvement of interchain transport is a promising route towards further enhancing the performance of coplanar polymer systems such as IDT-BT, and can be correlated to subbandgap absorption features. By contrast, the PDS spectrum of IDTT-BT does not contain this subbandgap charge transfer absorption feature and the Urbach energy is larger than that of IDT-BT. Consequently, IDTT-BT exhibits greater energetic disorder than IDT-BT and the absence of additional interchain charge transfer leads to the lower hole mobility that can be achieved in this system.

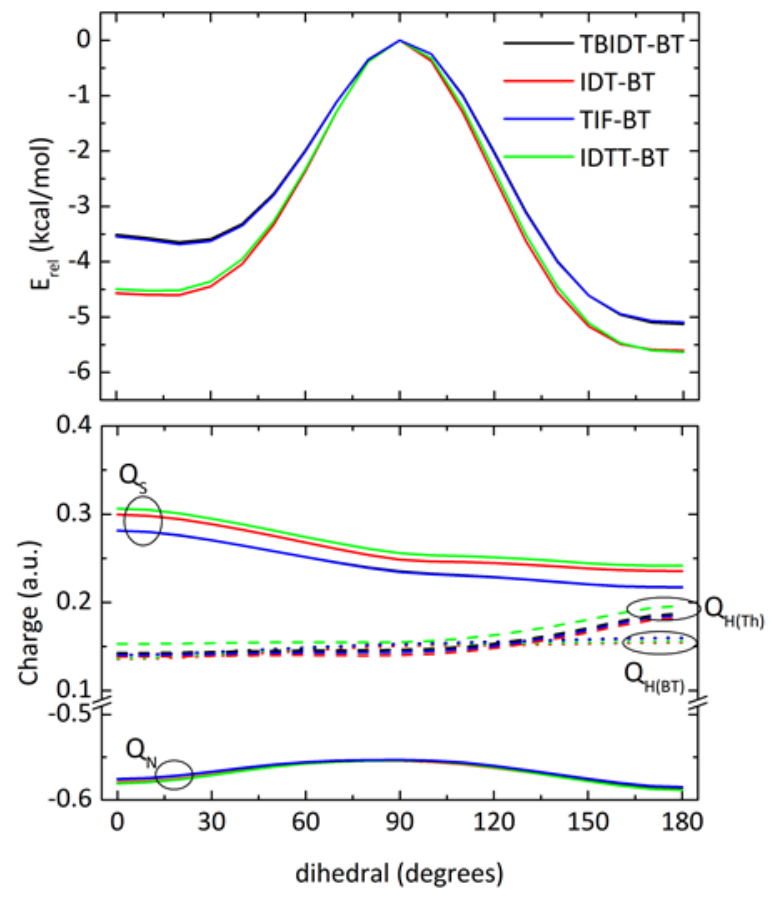

Figure 13. (Top) Computed torsional PES of extended IDT-BT derivatives, calculated at B3LYPD3/6-31G* level in gas phase. (Bottom) Mulliken partial charges on atoms involved in intramolecular non-covalent interactions as a function of dihedral angle.
A likely source for the observed energetic disorder is the variation in torsional conformation along the polymer backbone. To investigate, torsional PES were calculated for different extended IDT cores bearing a BT group (Figure 13). All extended IDTs show the same global minimum at the $180^{\circ}$ conformation, where non-traditional N-H hydrogen bonding can occur. Moreover, while IDTT shows almost identical energetics upon bond rotation to IDT, TIF and TBIDT groups differ by having slightly shallower minima, indicating less stabilization through non-covalent interactions. This also results in a slightly lower barrier for rotation, which may help to explain the increased torsional disorder in the polymers containing these groups.

The difference in PES is perhaps surprising, given that the non-covalent interactions are expected to be the same in each case, where a thiophene ring is bonded to the BT group. The IDT derivatives also all differ from the simpler thiophene model shown in Figure 7. Since the torsional potential depends largely on electrostatic forces, the partial charges of the atoms involved were extracted at each geometry of the PES scan (Figure 13). Firstly, it should be noted that the partial charge varies upon rotation as the electronic delocalization varies, and the atoms come close in space to other atoms. It is also observed that the partial charges on the BT atoms (nitrogen and hydrogen) are the same regardless of the IDT group. However, the partial charges on sulfur of the IDT group become less positive as the core is extended, while changes to the IDT hydrogen charge are more subtle. This should result in slightly weaker electrostatic stabilization with increasing IDT core size.

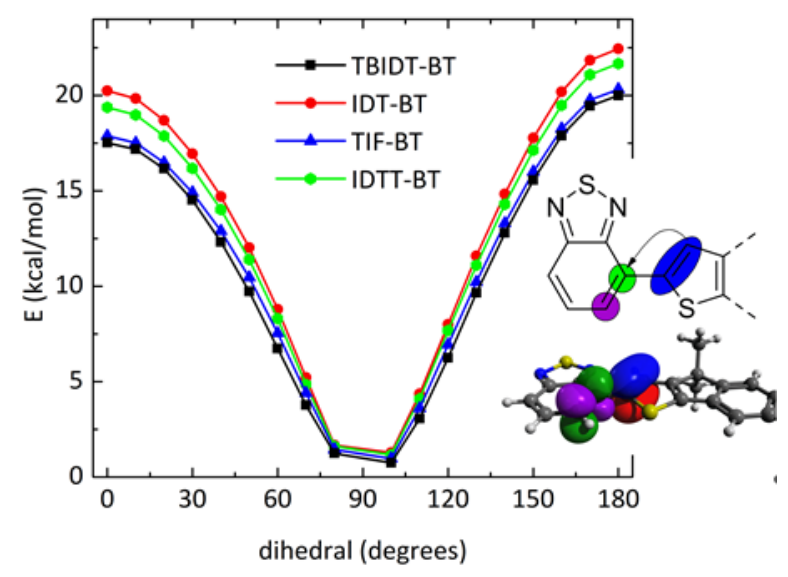

Figure 14. Resonance stabilization energies from natural bond orbital (NBO) delocalization across the rotatable bond, as a function of dihedral angle. Electrons are donated from a $\pi$ bond of IDT (red/blue) to the $\pi^{*}$ antibond in BT (green/purple). 
Another consequence to core extension is the change in electronic delocalization. To investigate this, NBO calculations on all optimized geometries from the PES were carried out, and the stabilization gained from conjugation across the rotatable bond was monitored. This comes from the energy of delocalization of the $\mathrm{C}=\mathrm{C} \pi$ bond of the IDT core with the $\mathrm{C}=\mathrm{C}^{*} \pi$ antibond of the $\mathrm{BT}$ group. At $90^{\circ}$, there is no conjugation between the two groups, and so this stabilization is zero. As the bond is rotated in either direction, the stabilization increases, with the $180^{\circ}$ conformer becoming more stabilized than the $\mathrm{O}^{\circ}$ one, as a consequence of a trans- alignment of the double bonds. The stabilization is in the order IDT $>$ IDTT $>$ TIFBT $>$ TBIDTBT and shows clear similarities to the overall PES plots.

\section{CONCLUSIONS AND OUTLOOK}

To summarize, a number of modifications to the structure of IDT-BT have been explored, in order to improve its hole mobility in OTFT devices. The choice of solubilizing alkyl chain length was found to be significant, dictating the polymer molecular weight that can be achieved, and influencing the degree of aggregation in the solid-state. The use of n-hexadecyl chains were found to strike the optimal balance of the solubility and aggregation properties necessary to form long polymer chains that are also able to form short contacts. Substitution of the carbon-bridgehead position of the IDT unit, to increase the planarity and increase the short contacts between polymer chains, was found not to be an effective method of improving the hole mobility in IDT-BT based polymers. Despite both the Si and Ge analogues exhibiting a greater degree of order in the solid state, neither were able to exceed hole mobilities of $0.05 \mathrm{~cm}^{2} \mathrm{~V}^{-}$ ${ }^{1} \mathrm{~s}^{-1}$. Whilst the reason for this is not entirely clear, it is likely that factors such as grain boundary limitations, misalignment of polymer lamellae, or lower polymer molecular weights may contribute. Moreover, it is evident that the use of the BT unit plays a pivotal role in the exceptional OTFT p-type mobilities achieved in the amorphous IDT based polymers. A highly ordered and planar polymer backbone is achieved as a result of favourable non-covalent interactions that occur between the heteroatom lone-pairs (on the IDT and BT) with the antibonding orbitals of the adjacent monomer, along with electrostatic interactions.

The most successful modifications to improve upon the hole mobility of IDT-BT involve extension of the IDT unit. Whilst this appeared to disrupt the backbone planarity of polymer chains,
TBIDT-BT and TIF-BT were able to compensate for this with improved interchain charge transfer, as observed from their PDS spectra. In the case of TIF-BT, only minor disruption to the backbone order, coupled with improved interchain charge transfer, resulted in the achievement of an OTFT hole mobility of $2.9 \mathrm{~cm}^{2} \mathrm{~V}^{-1} \mathrm{~s}^{-1}$, the only modified IDT copolymer to exceed the reference IDT-BT performance. This highlights that extension of the $\pi$-conjugated system may be among the most promising modifications to achieve high mobilities in these relatively amorphous IDT-based polymers.

\section{ASSOCIATED CONTENT}

Supporting Information. General experimental methods and GIWAXS linecuts of IDT-BT, TBIDT-BT and TIF-BT.

\section{AUTHOR INFORMATION}

\author{
Corresponding Authors \\ * Iain McCulloch \\ iain.mcculloch@imperial.ac.uk
}

\section{Author Contributions}

The manuscript was written through contributions of all authors. All authors have given approval to the final version of the manuscript. A. Wadsworth and $\mathrm{H}$. Chen contributed equally and share first authorship.

\section{ACKNOWLEDGMENT}

The authors thank KAUST for financial support and acknowledge EC FP7 Project SC2 (610115), EC H2O2O (643791), and EPSRC Projects EP/Go37515/1, $\mathrm{EP} / \mathrm{MOO} 5143 / 1$, and EP/LO16702/1. AS and CC gratefully acknowledge financial support from the $\mathrm{Na}-$ tional Science Foundation, Division of Materials Research Award\# 1808401. Use of the Stanford Synchrotron Radiation Lightsource, SLAC National Accelerator Laboratory, is supported by the U.S. Department of Energy, Office of Science, Office of Basic Energy Sciences under Contract No. DE-ACo276SF00515. The authors would also like to thank Aditya Sadhanala for providing the PDS data on IDTTBT.

\section{REFERENCES}

(1) Sirringhaus, H, Adv. Mater. 2014, 26, 13191335.

(2) Paterson, A. F.; Singh, S.; Fallon, K. J.; Hodsen, T.; Han, Y.; Schroeder, B. C.; Bronstein, H.; Heeney, M.; McCulloch, I.; Anthopoulos, T. D. Adv. Mater. 2018, 30, 1801079. 
(3) Yan, C.; Barlow, S.; Wang, Z.; Yan, H.; Jen, A. K. Y.; Marder, S. R.; Zhan, X. Nat. Rev. Mater. 2018, 3, 18003 .

(4) Geffroy, B.; le Roy, P.; Prat, C. Polym. Int. 2006, $55,572-582$.

(5) Crone, B.; Dodabalapur, A.; Lin, Y. Y.; Filas, R. W.; Bao, Z.; LaDuca, A.; Sarpeshkar, R.; Katz, H. E.; Li, W. Nature, 2000, 403, 521-523.

(6) de Leeuw, D. M.; Cantatore, E. Mat. Sci. Semicon. Proc.2008, 11, 199-204.

(7) Myny, K. Nat. Electron. 2018, 1, 30-39.

(8) Panidi, J.; Paterson, A. F.; Khim, D.; Fei, Z.; Han, Y.; Tsetseris, L.; Vourlias, G.; Patsalas, P. A.; Heeney, M.; Anthopoulos, T. D. Adv. Sci. 2017, 5, 1700290.

(9) Paterson, A. F.; Lin, H. Y.; Mottram, A. D.; Fei, Z.; Niazi, M. R.; Kirmani, A. R.; Amassian, A.; Solomeshch, Tessler, N.; Heeney, M.; Anthopoulos, T. D. Adv. Electron. Mater. 2017, 4, 1700464 .

(10) Soeda, J.; Okamoto, T.; Mitsui, C.; Takeya, J. Org. Electron. 2016, 39, 127.

(11) Kwon, S.; Kim, J.; Kim, G.; Yu, K.; Jo, Y. R.; Kim, B. J.; Kim, J.; Kang, H.; Park, B.; Lee, K.; Adv. Mater. 2015, 27, 6870.

(12)Sun, B.; Hong, W.; Yan, Z.; Aziz, H.; Li, Y. Adv. Mater. 2014, 26, 2636.

(13)Yang, J.; Zhao, Z.; Geneg, H.; Cheng, C.; Chen, J.; Sun, Y.; Shi, L.; Yi, Y.; Shuai, Z; Guo, Y.; Wang, S.; Liu, Y. Adv. Mater. 2017, 29, 1702115.

(14)Osaka, I.; Takimiya, K. Polymer, 2015, 59, A1A15.

(15) Tsao, H. N.; Cho, D.; Andreasen, J. W.; Rouhanipour, A.; Breiby, D. W.; Pisula, W.; Müllen, K. Adv. Mater. 2009, 21, 209-212.

(16)Kaake, L. G.; Barbara, P. F.; Zhu, X. Y. J. Phys. Chem. Lett. 2010, 1, 628-635.

(17)Caironi, M.; Bird, M.; Fazzi, D.; Chen, Z.; Di Pietro, R.; Newman, C.; Facchetti, A.; Sirringhaus, H. Adv. Funct. Mater. 2011, 21, 3371-3381.

(18)Schuettfort, T.; Watts, B.; Thomsen, L.; Lee, M.; Sirringhaus, H.; McNeill, C. R. ACS Nano, 2012, $6,1849-1864$.

(19)Liu, T.; Troisi, A. Adv. Funct. Mater. 2014, 24, 925-933.

(20) Sirringhaus, H. Adv. Mater. 2014, 26, 1319-1335.

(21) Sirringhaus, H.; Brown, P. J.; Friend, R. H.; Nielsen, M. M.; Bechgaard, K. Langeveld-Voss, B. M. W.; Spiering, A. H. H.; Janssen, R. A. J.; Meijer, E. W.; Herwig, P.; de Leeuw, D. M. Nature, 1999, 401, 685-688.

(22) McCulloch, I.; Heeney, M.; Bailey, C.; Genevicius, K.; MacDonald, I.; Shkunov, M.; Sparrowe, D.; Tierney, S.; Wagner, R.; Zhang, W.; Chabinyc, M. L.; Kline, R. J.; McGehee, M. D.; Toney, M. F. Nat. Mater. 2006, 5, 328-333.

(23) Zhang, X.; Bronstein, H.; Kronemeijer, A. J.; Smith, J.; Kim, Y.; Kline, R. J.; Richter, L. J.; Anthopoulos, T. D.; Sirringhaus, H.; Song, K.; Heeney, M.; Zhang, W.; McCulloch, I.; DeLongchamp, D. M. Nat. Commun. 2013, 4, 2238.

(24) Zhang, X. Richter, L. J,; DeLongchamp, D. M.; Kline, R. J.; Hammond, M. R.; McCulloch,
I.; Heeney, M.; Ashraf, R. S.; Smith, J. N.; Anthopoulos, T. D.; Schroeder, B.; Geerts, Y. H.; Fischer, D. A.; Toney, M. F. J. Am. Chem. Soc. 2011, 133, 15073-15084.

(25) Zhang, W.; Smith, J.; Watkins, S. E.; Gysel, R.; McGehee, M.; Salleo, A.; Kirkpatrick, J.; Ashraf, S.; Anthopoulos, T.; Heeney, M.; McCulloch, I. J. Am Chem. Soc. 2010, 132, 1143711439 .

(26) Venkateshvaran, D.; Nikolka, M.; Sadhanala, A.; Lemaur, V.; Zelazny, M.; Kepa, M.; Hurhangee, M.; Kronemeijer, A. J.; Pecunia, V.; Nasrallah, I.; Romanov, I.; Broch, K.; McCulloch, I.; Emin, D.; Oliver, Y.; Cornil, J.; Beljonne, D.; Sirringhaus, H. Nature, 2014, 515, 384-388.

(27) Kang, I.; Yun, H. J.; Chung, D. S.; Kwon, S. K.; Kim, Y. H. J. Am. Chem. Soc. 2013, 135, 14896-14899.

(28) Lei, T.; Dou, J. H.; Pei, J. Adv. Mater. 2012, 24, 6457-6461.

(29) Bronstein, H.; Leem, D. S.; Hamilton, R.; Woebkenburg, P.; King, S.; Zhang, W.; Ashraf, R. S.; Heeney, M.; Anthopoulos, T. D.; de Mello, J.; McCulloch, I. Macromolecules, 2011, 11, 6649-6652.

(30) Tsao, H. N.; Müllen, K. Chem. Soc. Rev. 2010, 39, 2372-2386.

(31) Scharber, M. C.; Koppe, M.; Gao, J.; Cordella, F.; Loi, M. A.; Denk, P.; Morana, M.; Egelhaaf, H. J.; Forberich, K.; Dennler, G.; Gaudiana, R.; Waller, D.; Zhu, Z.; Shi, X.; Brabec, C. J. Adv. Mater. 2010, 22, 367-370.

(32) Chen, H. Y.; Hou, J.; Hayden, A. E.; Yang, H.; Houk, K. N.; Yang, Y. Adv. Mater. 2010, 22, 371-375.

(33) Amb, C. M.; Chen, S.; Graham, K. R.; Subbiah, J.; Small, C. E.; So, F.; Reynolds, J. R. J. Am. Chem. Soc. 2011, 133, 10062-10065.

(34) Fei, Z.; Kim, J. S.; Smith, J.; Buchaca Domingo, E.; Anthopoulos, T. D.; Stingelin, N.; Watkins, S. E.; Kim, J. S.; Heeney, M. J. Mater. Chem. 2011, 21, 16257.

(35) Ohshita, J. Macromol. Chem. Phys. 2009, 210, 1360-1370.

(36) Ashraf, R. S.; Chen, Z.; Leem, D. S.; Bronstein, H.; Zhang, W.; Schroeder, B.; Geerts, Y.; Smith, J.; Watkins, S.; Anthopoulos, T. D.; Sirringhaus, H.; de Mello, J. C.; Heeney, M.; McCulloch, I. Chem. Mater. 2011, 23, 768-770.

(37) Fei, Z.; Ashraf, R. S.; Huang, Z.; Smith, J.; Kline, R. J.; D’Angelo, P.; Anthopoulos, T. D.; Durrant, J. R.; McCulloch, I.; Heeney, M. Chem. Commun. 2012, 48, 2955-2957.

(38) Donaghey, J. E.; Ashraf, R. S.; Kim, Y.; uang, Z. G.; Nielsen, C. B.; Zhang, W.; Schroeder, B.; Grenier, C. R. G.; Brown, C. T.; D’Angelo, P.; Smith, J.; Watkins, S.; Song, K.; Anthopoulos, T. D.; Durrant, J. R.; Williams, C. K.; McCulloch, I. J. Mater. Chem. 2011, 21, 18744-18752.

(39) Ashraf, R. S.; Schroeder, B. C.; Bronstein, H. A.; Huang, Z.; Thomas, S.; Kline, J. R.; Brabec, C. J.; Rannou, P.; Anthopoulos, T. D.; Durrant, J. R.; McCulloch, I. Adv. Mater. 2013, 25, 2029-2034. 

W.; Bronstein, H.; Ashraf, R. S.; Heeney, M.; McCulloch, I. Adv. Energy Mater. 2012, 2, 260265.

(41)Zhou, H.; Yang, L.; You, W. Macromolecules, 2012, 45, 607-632.

(42) Nielsen, C. B.; White, A. J. P.; McCulloch, I. J. Org. Chem. 2015, 80, 5045-5048.

(43) Nikolka, M.; Hurhangee, M.; Sadhanala, A.; Chen, H.; McCulloch, I.; Sirringhaus, H. Adv. Electron. Mater. 2018, 4, 1700410.

(44) Schroeder, B. C.; Huang, Z.; Ashraf, R. S.; Smith, J.; D'Angelo, P.; Watkins, S. E.; Anthopoulos, T. D.; Durrant, J. R.; McCulloch, I. Adv. Funct. Mater. 2012, 22, 1663-1670.

(45) Planells, M.; Nikolka, M.; Hurhangee, M.; Tuladhar, P. S.; White, A. J. P.; Durrant, J. R.; Sirringhaus, H.; McCulloch, I. J. Mater. Chem. C, 2014, 28789.

(46) Thorley, K. J.; McCulloch, I. J. Mater. Chem. C, 2018, 6, 12413.

(47) Jackson, N. E.; Savoie, B. M.; Kohlstedt, K. L.; de la Cruz, M. O.; Schatz, G. C.; Chen, L. X.; Ratner, M. A. J. Am. Chem. Soc. 2013, 135, $10475-10483$.

(48) Bronstein, H.; Frost, J. M.; Hadipour, A.; Kim, Y.; Nielsen, C. B.; Ashraf, R. S.; Rand, B. P.; Watkins, S.; McCulloch, I. Chem. Mater. 2013, 25, 277-285.

(49) Zhang, W.; Han, Y.; Zhu, X.; Fei, Z.; Feng, Y.; Treat, N. D.; Faber, H.; Stingelin, N.; McCulloch, I.; Anthopoulos, T. D.; Heeney, M. Adv. Mater. 2016, 28, 3922-3927.

(50) Chen, H.; Hurhangee, M.; Nikolka, M.; Zhang, W.; Kirkus, M.; Neophytou, M.; Cryer, S. J.; Harkin, D.; Hayoz, P.; Abdi-Jalebi, M.; McNeill, C. R.; Sirringhaus, H.; McCulloch, I. Adv. Mater. 2017, 29, 1702523.

(51) Thomas, T. H.; Harkin, D. J.; Gillet, A. J.; Lemaur, V.; Nikolka, M.; Sadhanala, A.; Richter, J. M.; Armitage, J.; Chen, H.; McCulloch, I.; Menke, M.; Olivier, Y.; Beljonne, D.; Sirringhaus, H. Nat. Commun. 2019, 10, 2614. 
Insert Table of Contents artwork here

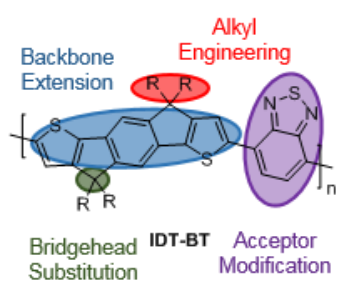

